\title{
Gestational Diabetes Mellitus in Pakistan: Undiagnosed Threat to Pregnant Women
}

\author{
Maria Tahir ${ }^{1}$, Noman ul Haq ${ }^{2}$, Arooj Abid ${ }^{3, *}$, Abeera Zafar ${ }^{2}$ \\ ${ }^{1}$ Department of Pharmacy, Sardar Bahadur Khan University, Quetta, PAKISTAN. \\ ${ }^{2}$ Department of Pharmacy Practice, University of Balochistan, Sariab Road, Balochistan, PAKISTAN. \\ ${ }^{3}$ Riphah Institute of Pharmaceutical Sciences, Riphah International University, Islamabad, PAKISTAN.
}

\section{Dear Editor,}

Gestational Diabetes Mellitus (GDM) is a rare disorder and due to non-uniformity in its diagnostic criteria and changes over time in the recommended glucose values ${ }^{[1]}$ it is a major and a severe problem during pregnancy. But the time has reached to draw peoples' attention towards this major medical complication of pregnancy. Women with risk factors such as age greater than 25 years; being overweight i.e., BMI $>27 \mathrm{~kg} / \mathrm{m}^{2}$ before pregnancy and BMI $>30 \mathrm{~kg} / \mathrm{m}^{2}$ during pregnancy; family history of diabetes; the previous history of pregnancy-induced hypertension and weight of the previous baby of $\geq 4.5 \mathrm{~kg}$ have a greater incidence of hyperglycemia during their pregnancy period. ${ }^{[2]}$

American Diabetes Association recommends that all pregnant women without a prior known history of diabetes should undergo a $75 \mathrm{~g}$ Oral Glucose Tolerance Test (OGTT) at 24-28 weeks of gestation with a purpose of improving gestational life and preventing adverse pregnancy outcomes. ${ }^{[3]}$ Women with GDM may have LGA (large for gestational age) for birth weight, stillbirth, difficult delivery, neonatal morbidities (respiratory difficulties, neonatal hypoglycemia, jaundice and in most cases death) and impaired healing of the surgical site after cesarean section as a result of uncontrolled diabetes during pregnancy. ${ }^{[4]}$

In this context, the health care system of Pakistan has no proper diagnostic test being performed for Gestational Diabetes Mellitus (GDM). Women are not advised to have the Oral Glucose Tolerance Test (OGTT) for the diagnosis of GDM. In most cases, patients are advised to get their plasma glucose level tested which is not a proper test for diagnosis of GDM as compared to OGTT or $\mathrm{HbA}_{1 c}$. As a result, timely, women with GDM are not diagnosed, the majority of pregnant women learn about their diabetes after giving birth to their child when either the complications appear in neonates or when their the wound gets infected. Moreover, women with GDM bear complications during delivery which lead to C-Section or even develop diabetes mellitus type 2 after the termination of pregnancy.

Considering the current practice in Pakistan regarding GDM and its diagnosis, it is highly recommended that all the pregnant women with any of the risk factors related to GDM should undergo OGTT and/or $\mathrm{HbA}_{1 \mathrm{c}}$ at the start of pregnancy and/or second trimester of pregnancy so that the complications can be avoided and GDM can be properly managed.

\section{ACKNOWLEDGEMENT}

All authors equally contributed in writing the correspondence.

\section{CONFLICT OF INTEREST}

We declare no competing interests.

\section{REFERENCES}

1. Ferrara A, Hedderson MM, Quesenberry CP, Selby JV. Prevalence of gestational diabetes mellitus detected by the national diabetes data group or the carpenter and coustan plasma glucose thresholds. Diabetes Care. 2002;25(9):1625-30.

2. Cheung NW, Wasmer G, Al-Ali J. Risk factors for gestational diabetes among Asian women. Diabetes Care. 2001;24(5):955-6.

3. Association AD. Diagnosis and classification of diabetes mellitus. Diabetes Care. 2014;37(Supplement 1):S81-90.

4. Salzer L, Yogev Y. Complications of gestational diabetes. Gestational Diabetes: Origins, Complications and Treatment. 2014:95-109.

Correspondence:

Dr. Arooj Abid

Riphah Institute of Pharmaceutical Sciences, Riphah International University, G-7/4, Islamabad, PAKISTAN.

Email: aroojaabid@gmail.com DOI: $10.5530 /$ ijcep.2019.4.20

Cite this article as: Tahir M, Haq N, Abid A, Zafar A. Gestational Diabetes Mellitus in Pakistan: Undiagnosed Threat to Pregnant Women. J Pharm Pract Community Med. 2019;5(4):80 